Einführung zum Thema

Pneumologe 2019 · 16:331-332

https://doi.org/10.1007/s10405-019-00273-w

c) Springer Medizin Verlag GmbH, ein Teil von Springer Nature 2019

Wer kann sich nicht an die therapeutische Hilflosigkeit von Ärzten beim nichtkleinzelligen Lungenkarzinom („non-small cell lung cancer", NSCLC) IV vor 30 Jahren erinnern? Und welche Erleichterung war zu spüren, nachdem die Analyse der NSCLC Collaborative Group im BMJ 1995 die wissenschaftliche Evidenz erbrachte, dass eine zytostatische Chemotherapie bei metastasierten NSCLC einen positiven Effekt auf die Überlebenszeit hatte: Verbesserung der Einjahresüberlebensrate um $10 \%$, und der medianen Überlebenszeit um 1,5 Monate, entsprechend einer Hazard Ratio von 0,73 bzgl. des Sterberisikos [1].

\section{》) Eine heutzutage als Standard anzusehende molekulare Thera- piestratifizierung setzt eine High- tech-Molekularpathologie voraus}

Die Überschrift „Lungenkarzinom: Präzise Diagnostik rettet Leben“" in der Rubrik "Medizinreport“ im Deutschen Ärzteblatt [2] weist auf die pathologischen Erkenntnisse hin, welche die Grundlage der modernen Therapie des NSCLC IV geworden sind. Neben der klassischen Pathologie hat sich nicht nur eine Subspezialität in der Pathologie entwickelt, sondern es hat sich ein der klassischen Pathologie gleichwertiger Arbeitsbereich etabliert, welcher nunmehr die entscheidende Determi-

\title{
Dieter Ukena
}

Klinik für Pneumologie und Beatmungsmedizin, Lungenzentrum am Klinikum Bremen-Ost, Klinikum Bremen-Ost, Bremen, Deutschland

\section{Fortgeschrittenes Lungenkarzinom - Prognoseverbesserung durch zielgerichtete Therapiemodalitäten!}

nante der pathologischen Untersuchung von Lungentumoren darstellt: die Molekularpathologie. Eine heutzutage als Standard anzusehende molekulare Therapiestratifizierung setzt eine HightechMolekularpathologie voraus, die nur in wenigen spezialisierten Institutionen geleistet werden kann. In Ergänzung zur klassischen Pathologie inkl. der Immunhistochemie sind molekularpathologische Untersuchungen notwendig, die eher von Molekularbiologen als von konventionellen Pathologen geleistet werden kann.

Die molekulare Diagnostik von Lungentumoren und die konsekutive Therapieplanung soll sinnvollerweise in spezialisierten Diagnostikzentren zentralisiert werden. Das ist die Idee, die dem von Prof. R. Büttner und Prof. J. Wolf (Universität Köln) mit Unterstützung der Deutschen Krebshilfe geförderten nationalen Netzwerk Genomische Medizin (nNGM) zugrunde liegt. Im 2018 gegründeten NGM sind 15 universitäre Krebszentren vereint, welche eine zentrale Diagnostik und Therapieplanung für Patienten aus dem ganzen Bundesgebiet anbieten.

Wie komplex die molekulare Pathologie geworden ist, wird aus dem Beitrag von Markus Falk et al. aus dem Lungennetzwerk NOWEL (Hamburg) zur „Molekularpathologie des Lungenkarzinoms: aktuelle Standards und weitere Entwicklungen" deutlich. Einige Stichworte aus dem Komplex Molekularpathologie: - molekulare Therapiestratifizierung,
- Next Generation Sequencing (NGS),

- Treibermutationen (EGFR, ALK, ROS1, BRAF, RET, Exon14 skipping MET, NTRK),

- Immuntherapie: PD-L1 als Biomarker,

- Tumormutationslast (TMB) als Biomarker,

- Liquid Biopsy.

Der Beitrag von M. Falk et al. (Hamburg) ist auch ein Beispiel dafür, wie komplexe Pathologie auf didaktisch überzeugende Art und Weise einem "normalen Arzt" nähergebracht werden kann.

In dem Beitrag von W.M. Brückl \& J.H. Ficker (Nürnberg) „Metastasiertes Lungenkarzinom - therapierbare molekulare Alterationen" werden nicht nur die Wertigkeit der prätherapeutischen Mutationsanalyse dargestellt und klare Aussagen zur Auswahl von Tyrosinkinase-Inhibitoren (TKI) getroffen, sondern auch das Prozedere im Fall einer Resistenzentwicklung erklärt:

- Eine erneute Mutationsanalyse wird durchgeführt, am besten in einer Gewebeprobe, die am Ort des Progresses entnommen wurde.

- Falls eine erneute Gewebebiopsie nicht möglich ist, erfolgt die DNA-/ RNA-Analyse im Blut (sog. Liquid Biopsy).

- Bei Nachweis der molekularen Alterationen, für die aktuell kein Medikament zugelassen ist, sollte versucht werden, den Patienten in 
eine klinische Studie einzuschließen (s. Ausführung zum nationalen Netzwerk Genomische Medizin, nNGM, in diesem Editorial).

- Im Fall einer Krankheitsprogression nach EGFR-TKI und bei fehlendem Nachweis einer therapierbaren Resistenzmutante Einsatz der sog. Quadrupel-Therapie bestehend aus Atezolizumab, Bevacizumab und Carboplatin/Paclitaxel.

Der mit Abstand größte Hype in der Onkologie des zurückliegenden Jahrzehnts wurde durch die Immuntherapie mit Einsatz sog. Checkpointinhibitoren ausgelöst. Im wahrsten Sinne gekrönt wurde die Therapie mit Immunonkologika (IO) durch die Verleihung des Medizinnobelpreises 2018 an James P. Allison (USA) und Tasuku Honjo (Japan). Dabei ist ein Ende der Reise mit IO in der Krebstherapie noch gar nicht absehbar. Nahezu jede Organkrebsentität wird mittlerweile mit IO behandelt.

In dem Beitrag von S. Sackmann et al. (Bremen) über „Immuntherapeutika in der Erstlinientherapie des metastasierten Lungenkarzinoms" werden die vielfältigen Einsatzmöglichkeiten der PD-1-/PD-L1-Inhibitoren allein oder in Kombination mit Zytostatika oder anderen Biologika wie Angiogenese-Inhibitoren dargestellt. Es werden auch die Unterschiede zwischen den verschiedenen Checkpointinhibitoren verdeutlicht. Schließlich wird kritisch das Einsatzgebiet der Checkpointinhibitoren in der Erstlinientherapie des NSCLC IV beleuchtet - zur Beantwortung der Frage „Immuntherapie für alle?“.

\section{》) Ein neues Indikationsgebiet für Immuntherapeutika ist das lokal fortgeschrittene, inoperable NSCLC}

Ein neues Indikationsgebiet für Immuntherapeutika ist das lokal fortgeschrittene, inoperable NSCLC (also Stadium III). In dem Beitrag von A. Fabian et al., (Kiel) „Lokal fortgeschrittenes nichtkleinzelliges Lungenkarzinom: Radioimmuntherapie als neuer Standard?" werden die An- haltspunkte für die immunaktivierende Wirkung einer lokalen Strahlentherapie (inkl. des abskopalen Effekts) und für die synergistische Wirkung zwischen IO und Radiatio verdeutlicht. Beim inoperablen NSCLC III ist die Immunerhaltungstherapie mit Durvalumab nach kombinierter platinhaltiger Chemo-/Radiotherapie der neue Therapiestandard - mit signifikanter und klinisch relevanter Verbesserung der Prognose. Zahlreiche Studien beschäftigen sich mit der Kombination Strahlentherapie/Immuntherapie - und es wird sicherlich spannend werden bei der Beurteilung einer derartigen Kombination mit den Ergebnissen der chirurgischen Resektionsverfahren.

Ungefähr $15 \%$ der Lungenkarzinome weisen die pathologischen Charakteristika eines neuroendokrinen Tumors auf, des kleinzelligen Lungenkarzinoms (SCLC, „small cell lung cancer“). In der Regel wird ein SCLC im metastasierten Stadium diagnostiziert, sodass kein kurativer Therapieansatz besteht. Tatsache ist, dass in den zurückliegenden 2-3 Jahrzehnten praktisch keine Fortschritte in der Therapie des metastasierten SCLC erzielt wurden. Wie Monika Serke (Hamm) in ihrem wirklich informativen Beitrag „Kleinzelliges Lungenkarzinom: Neue Hoffnung auf eine Präzisionsmedizin mit Chemoimmuntherapie nach 30 Jahren therapeutischen Stillstands?" darstellt, hat eine intensive Suche nach Biomarkern eingesetzt auch hier mit der Perspektive, eine zielgerichtete medikamentöse Systemtherapie zu entwickeln. Mögliche Zielstrukturen sind PARP (ADP-Ribose-Polymerase), EZH2 („enhancer of zeste homologue 2“) oder DLL3 („delta-like canonical Notch ligand 3“), Wirkstoffe wie z.B. Olaparib oder Rova-T werden in klinischen Studien untersucht. Letztendlich gab es auch Fortschritte in der Zytostatika + IO-Kombinationstherapie. Der PDL1-Inhibitor Atezolizumab plus Carboplatin/Etoposid mit AtezolizumabErhaltungstherapie erhielt in Europa eine Zulassung für die Erstlinientherapie des metastasierten SCLC. Allerdings „tut sich die Immuntherapie noch schwer" beim SCLC: längst nicht alle Studien mit IO waren positiv, und die erzielten Effekte waren nicht vergleichbar mit denjenigen beim NSCLC. Nun denn, es besteht für Raum für Verbesserung!

Die Fortschritte insbesondere in Form der molekular stratifizierten Therapie des Lungenkarzinoms sind atemberaubend. Umso mehr die Verpflichtung für uns Lungenärzte, auf der Höhe dieser Entwicklungen $\mathrm{zu}$ bleiben und $\mathrm{zu}$ versuchen, unseren schwerkranken Patienten die bestmögliche Therapie anzubieten.

Viel Freude bei der Lektüre dieser Ausgabe von Der Pneumologe wünscht Ihnen

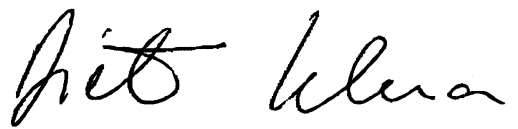

Univ.-Prof. Dr. Dieter Ukena (Bremen)

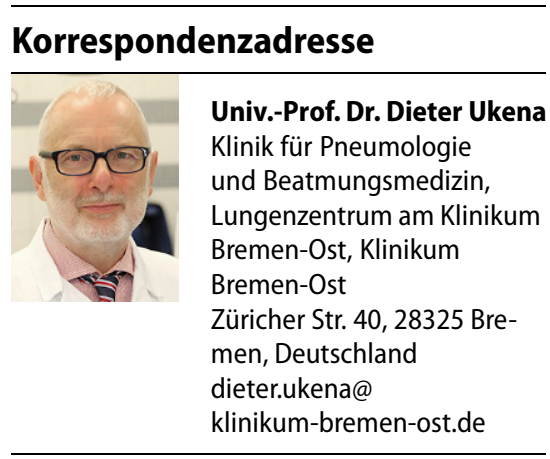

Interessenkonflikt. D. Ukena weist auf folgende Beziehungen hin: Erhalt von Honoraren aus Referententätigkeit von den Firmen Amgen, AstraZeneca, Boehringer Ingelheim, Bristol Myers Squibb, MSD, Roche. Erhalt von Honoraren für Beratertätigkeit von AstraZeneca, Boehringer Ingelheim, MSD, Takeda.

\section{Literatur}

1. Non-Small Cell Lung Cancer Collaborative Group (1995) Chemotherapy in non-small cell lung cancer: A meta-analysis using updated data on individual patients from 52 randomized trials. BMJ 311:899. https://doi.org/10.1136/bmj.311.7010. 899

2. Eckert N (2019) Lungenkarzinom: Präzise Diagnostik rettet Leben. Dtsch Arztebl 116(29-30):A-1377 / B-1138/C-1122 